EXTENDED REPORT

\section{Silicone oil concentrates fibrogenic growth factors in the retro-oil fluid}

\author{
R H Y Asaria, C H Kon, C Bunce, C S Sethi, G A Limb, P T Khaw, G W Aylward, D G Charteris
}

Br J Ophthalmol 2004;88:1439-1442. doi: 10.1136/bjo.2003.040402

See end of article for
authors' affiliations
$\ldots \ldots \ldots \ldots \ldots \ldots \ldots \ldots \ldots$
Correspondence to:
Mr David Charteris,
Vitreoretinal Research
Unit, Moorfields Eye
Hospital, City Road,
London EC1V 2PD, UK;
david.charteris@
moorfields.nhs.uk
Accepted for publication
1 April 2004

See end of article for authors' affiliations

Correspondence to: Mr David Charteris, Vitreoretinal Research Unit, Moorfields Eye London ECIV 2PD, UK david.charteris@ 1 April 2004 ...................
S ince the early use of silicone oil for the treatment of complex retinal detachment ${ }^{1}$ there have been concerns about its potential retinal toxicity. ${ }^{23}$ In general, these have not been substantiated by clinical experience and silicone oil has been used increasingly for the anatomical reattachment of the retina in patients with complex retinal detachments associated with proliferative diabetic retinopathy, ${ }^{45}$ proliferative vitreoretinopathy (PVR), ${ }^{6}$ giant retinal tears, ${ }^{7}$ and trauma. ${ }^{8}$

The potential of silicone oil to promote a proliferative response within the eye has been an additional concern and Lewis $^{9}$ has observed that silicone oil appears to enhance cellular proliferation and formation of preretinal membranes in eyes treated for advanced PVR. Subsequent reports have also noted an incidence of perisilicone proliferation. ${ }^{10}$ An analysis of PVR epiretinal membranes has also shown that membranes from eyes with silicone oil contain T lymphocytes in contrast with membranes from eyes without silicone oil which had no T lymphocytes, ${ }^{11}$ suggesting that an inflammatory reponse to silicone oil may contribute to fibrosis.

These clinical observations have been supported by analysis of animal models. ${ }^{12}{ }^{13}$ In an experimental study Lambrou and co-workers ${ }^{14}$ reported that silicone oil increases the risk of experimental PVR using an in vitro proliferation assay and also demonstrated that vitreous cavity silicone oil had increased mitogenic activity for retinal pigment epithelial cells compared to gas filled or fluid filled vitreous. They reasoned that silicone oil appears to increase proliferation by stimulating the release of more or different mitogenic factors as well as potentially concentrating active factors into a smaller volume near the retina.

This study was designed to investigate the presence of growth factors potentially able to contribute to the formation of fibrocellular membranes in the fluid surrounding silicone oil in human eyes that have undergone vitreoretinal surgery. We compared this (retro-oil) fluid with vitreous cavity fluid from patients undergoing revision vitrectomy surgery and with vitreous from patients undergoing primary vitrectomy surgery for (a) macular hole and (b) primary retinal detachment with and without PVR. We analysed the fibrogenic growth factors transforming growth factor beta $\left(\mathrm{TGF}-\beta_{2}\right.$ ) and basic fibroblast growth factor (bFGF) and the inflammatory cytokine interleukim 6 (IL-6), together with total protein levels. Where appropriate statistical comparison was also carried out on the differing control samples.

\section{PATIENTS AND METHODS}

The study was conducted in accordance with the principles embodied in the Declaration of Helsinki and informed consent was obtained from all patients after the nature of the study was explained.

Three hundred and nine patients undergoing vitreoretinal procedures at Moorfields Eye Hospital (see table 1) were prospectively enrolled in the study from 1995 to 1997. Retrooil fluid samples were taken from phakic patients undergoing removal of silicone oil. Preoperative PVR was considered to be present if 1 clock hour or more of grade C PVR was present according to the Retinal Society PVR classification. ${ }^{15}$ None of the patients recruited had a previous history of diabetic retinopathy, uveitis, or other ocular pathology which could influence levels of protein or growth factors in the posterior segment. Medical grade silicone oil (Dow-Corning), 1000 centistokes, was used in all eyes which had silicone oil tamponade.

\section{Collection of vitreous}

At the beginning of surgery, before commencing intraocular infusion, an undiluted sample of vitreous, retro-oil fluid, or vitreous cavity fluid was obtained. To obtain retro-oil fluid a 20 gauge cannula attached to a syringe was introduced into the fluid behind the oil. Fluid was then aspirated to obtain a sample of approximately $500-750 \mu \mathrm{l}$. In the other cases

Abbreviations: bFGF, basic fibroblast growth factor; ELISA, enzyme linked immunosorbent assays; IL, interleukin; PVR, proliferative vitreoretinopathy; TGF- $\beta_{2}$, transforming growth factor beta 
Table 1 Patient groups, growth factor, and protein levels

\begin{tabular}{|c|c|c|c|c|}
\hline & TGF- $\beta_{2}$ (ng/ml) & B-FGF (pg/ml) & Il-6 (pg/ml) & Protein $(\mathrm{mg} / \mathrm{ml})$ \\
\hline Sample type & Median (IQR) & Median (IQR) & Median (IQR) & Median (IQR) \\
\hline Retro-oil fluid $(n=13)$ & $1.65(1.03-2.30)$ & $50.85(28.46-60.48)$ & $572.18(523.81-581.37)$ & $736.32(343.63-1368.56)$ \\
\hline Macular hole vitreous $(n=10)$ & $1.07(0.65-1.90)$ & $0.18(0.15-1.76)$ & $0.98(0.9-1.43)$ & $20.60(8.95-49.83)$ \\
\hline $\begin{array}{l}\text { Revision-vitrectomy fluid } \\
(\mathrm{n}=11)\end{array}$ & $1.24(0.93-1.78)$ & $2.14(1.05-2.48)$ & $515.18(78.38-615.08)$ & $149.22(46.77-593.8)$ \\
\hline $\begin{array}{l}\text { No preoperative PVR vitreous } \\
(n=244)\end{array}$ & $1.54(0.70-3.13)$ & $1.99(0.72-10.77)$ & $106.88(31.28-384.23)$ & $75.5(30-151)$ \\
\hline $\begin{array}{l}\text { Preoperative PVR vitreous } \\
(n=31)\end{array}$ & $1.70(1.03-2.85)$ & $13.21(5.11-59.19)$ & $296.93(63.0-628.58)$ & $218(112-706.92)$ \\
\hline $\begin{array}{l}\text { Non retro-oil samples } \\
\text { combined }\end{array}$ & $1.51(0.79-3.1)$ & $2.23(0.77-12.63)$ & $110.1(29.18-418.65)$ & $81.5(30.415-173.98)$ \\
\hline
\end{tabular}

undiluted vitreous or vitreous cavity fluid samples were collected by aspiration through the vitreous cutter. Undiluted samples were divided into aliquots in siliconised tubes (Eppendorf, Freemont, CA, USA) and kept frozen at $-70^{\circ} \mathrm{C}$ until each analysis.

\section{Enzyme linked immunosorbent assays (ELISA)}

Levels of TGF- $\beta 2$, bFGF, and IL-6 were analysed using sandwich enzyme immunoassay kits (R\&D Systems, Oxon, UK). Pilot studies were performed to determine the appropriate dilutions for each growth factor. The sample volumes used were $200 \mu \mathrm{l}$ (1:24 dilution) for TGF- $32,150 \mu \mathrm{l}$ (1:15 dilution) for bFGF, and $200 \mu \mathrm{l}$ (1:75 dilution) for IL-6. The minimum detectable concentrations (sensitivity) for the assay kits were $2.00 \mathrm{pg} / \mathrm{ml}, 0.043 \mathrm{pg} / \mathrm{ml}$, and $0.08 \mathrm{pg} / \mathrm{ml}$ for TGF- $\beta 2$, bFGF and IL- 6 respectively. Since the assay for TGF$\beta 2$ only detects its active form, samples to be analysed for this factor were first activated by the addition of $1 \mathrm{M} \mathrm{HCl}(40 \mu \mathrm{l}$ $\mathrm{HCl} / 200 \mu \mathrm{l}$ sample). These were then neutralised with $40 \mu \mathrm{l}$ $1.2 \mathrm{M} \mathrm{NaOH} / 0.5 \mathrm{M}$ HEPES. The assays therefore measured the total amount of potentially active TGF- $\beta 2$.

\section{Protein analysis}

The total protein concentrations of the samples were measured using a commercial assay (Protein Microassay; BioRad, Herts, UK). This colorimetric assay is a solution of cupric ions that forms a copper-protein complex (coloured compound) with protein. It allows rapid screening of multiple small volume fluid and vitreous samples.

\section{Statistical analysis}

Growth factor levels were compared between retro-oil fluid and all other vitreous and vitreous fluid samples individually using the Wilcoxon rank sum (Mann-Whitney) test. The retro-oil fluid samples were also compared with the control specimens combined. Selected comparison of control specimens was carried out where these were considered to be biologically relevant: revision vitrectomy fluid was compared to PVR and to non-PVR vitreous and PVR vitreous was compared to non-PVR vitreous. Non-parametric methods were used because the data showed considerable skewness. No adjustment was made for multiple testing because the analysis was hypothesis generated. Statistical calculations were performed using commercial software (Stata, StataCorp. 2003. Stata Statistical Software: Release 8.0. College Station, TX, USA: StataCorp LP)

\section{RESULTS}

Complete data were available for all patients. Of the 13 patients undergoing removal of silicone oil from whom retrooil fluid was obtained two had had previous (preoperative)
PVR and seven had evidence of new proliferation (postoperative) PVR before oil removal. In these patients the duration of silicone oil tamponade ranged from $\mathrm{l}$ to 26 months with a median of 3 months. Two patients had oil in situ for longer than 6 months but their protein and growth factor levels were not markedly different from those with lesser durations. All 13 retro-oil fluid patients had had silicone oil injected because of clinical features suggestive of a high risk of postoperative PVR development: two had existing PVR, two had had previous retinal detachment surgery, one had a giant retinal tear, and the others had large or multiple retinal breaks.

In the revision vitrectomy fluid group three of 11 cases had PVR; 34 of the total of 298 non-retro-oil control samples had PVR.

The median levels of growth factors and protein for the various groups are documented in table 1 . The probability values for the statistical analyses of comparisons of medians using the Mann-Whitney test are presented in table 2. The median levels of bFGF and IL-6 in the retro-oil fluid were significantly raised compared to all the other vitreous and vitreous fluid samples except vitreous from eyes with PVR (table 2) and the IL-6 value in revision vitrectomy. Protein in retro-oil fluid was raised in comparison with all other specimens. There was little evidence of any difference, however, between the median level of TGF- $\beta_{2}$ in the retrooil fluid compared to the other samples (tables 1 and 2).

PVR vitreous had raised levels of bFGF, IL-6, and protein but not TGF- $\beta_{2}$ when compared to non-PVR vitreous (tables 1 and 2). Levels of growth factors and protein in revision vitrectomy fluid showed little difference from vitreous from eyes with or without PVR apart from a significantly raised level of bFGF in eyes with PVR (tables 1 and 2).

\section{DISCUSSION}

This study demonstrates that two fibrogenic cytokines (bFGF and IL-6) and total protein levels are significantly raised in the retro-oil fluid compartment compared to vitreous from eyes with macular holes and retinal detachment without PVR and compared to vitreous cavity fluid from eyes undergoing revision vitrectomy. Total protein was also raised when analysed against vitreous from eyes with PVR. Selecting the optimal control specimens for the comparison of retrosilicone oil fluid is problematic. Vitreous from eyes with retinal detachment, with and without PVR, or with macular hole serves as a useful measure of growth factors levels in more "normal" situations in the eye. The use of formed vitreous itself could, however, produce a bias both in the intraocular distribution of protein molecules and in the ELISA assay. We have therefore additionally used vitreous cavity fluid from eyes undergoing revision vitrectomy surgery 
Table 2 Probability (p) values for comparisons of specimens (Wilcoxon rank sum (MannWhitney) test)

\begin{tabular}{lllll}
\hline Comparison & TGF- $\beta$ & bFGF & IL-6 & Protein \\
\hline Retro-oil $v$ macular hole & 0.24 & 0.002 & 0.0001 & 0.0001 \\
Retro-oil $v$ revision vitrectomy & 0.54 & 0.0007 & 0.32 & 0.005 \\
Retro-oil $v$ non-PVR vitreous & 0.76 & 0.0001 & 0.0003 & $<0.0001$ \\
Retro-oil $v$ PVR vitreous & 0.72 & 0.063 & 0.052 & 0.015 \\
PVR vitreous $v$ non-PVR vitreous & 0.27 & 0.0004 & 0.035 & $<0.0001$ \\
Revision vitrectomy $v$ non-PVR vitreous & 0.86 & 0.69 & 0.086 & 0.17 \\
Revision vitrectomy $v$ PVR vitreous & 0.29 & 0.0027 & 0.92 & 0.18 \\
Retro-oil $v$ combined control specimens & 0.75 & 0.0002 & 0.0005 & $<0.0001$ \\
\hline
\end{tabular}

to eliminate this potential bias. The use of a number of control specimens from differing conditions strengthens the argument that the statistically significant differences seen in growth factor and protein levels in retro-oil fluid represent a real pathobiological alteration in their concentration. The consistency of the results of growth factor and protein analyses between control specimens (and for the individual growth factors, see tables 1 and 2) is further evidence that the controls used are valid.

An effect of the underlying pathology on growth factor levels cannot be discounted, again the use of multiple controls helps to define the role of silicone oil in the altered growth factor concentrations we have demonstrated. It is notable that levels of growth factors were not significantly different in retro-oil fluid from PVR vitreous suggesting that the presence of PVR (seen in seven of 13 retro-oil fluid specimens) has an important influence on growth factor pathobiology.

Basic FGF has been shown to enhance the proliferation of Muller cells, retinal astrocyes, and retinal pigment epithelial cells in vivo, ${ }^{16}$ and has previously been shown to be elevated in the vitreous of eyes developing $\mathrm{PVR}^{17-19}$ (a similar finding to that of the analysis of controls in this study) and clearly has the potential to have a role in mediating the proliferative and fibrogenic responses in PVR. Raised levels in the intraocular fluid surrounding silicone oil point to a potential similar role in perisilicone proliferation.

IL-6 is an important mediator of the acute phase reaction in inflammatory and immune responses. Cells with IL-6 mRNA expression have been found in PVR epiretinal membranes ${ }^{20}$ and previous work has also have shown that the vitreous levels of IL- 6 are significantly raised in vitreous samples obtained from patients with PVR. ${ }^{18} 1921$ The consistent finding of raised IL-6 levels is evidence for its role in the marked blood-retinal barrier breakdown seen in PVR.

Both basic FGF and IL-6 have the potential to contribute to the formation of fibrocellular epiretinal membranes and the concentration of these growth factors found in the retrosilicone oil fluid points to a possible pathogenic mechanism for perisilicone membrane formation. It is notable that animal studies have demonstrated silicone oil can have an enhanced mitogenic effect on RPE cells and an increased PVR rate in silicone filled vitreous. ${ }^{14}$

The present investigation has additionally shown that there is an increase in total protein in the retro-oil fluid. This may be a result of a concentration effect caused by the enhanced inflammatory response and blood-retinal barrier breakdown seen in eyes that will generally have an existing predisposition to fibrogenic proliferation. An increased total protein level may represent a marker of the concentration of inflammatory and fibrogenic mediators in the retro-oil fluid milieu that could be central to the reproliferation which can complicate complex retinal reattachment surgery. However, it is notable that, compared to control samples, TGF- $\beta$ levels were not raised in the retro-oil fluid (or in the PVR vitreous or revision vitrectomy fluid). This suggests that the increased levels of bFGF and IL- 6 may be the result of enhanced active secretion rather than a more generalised concentration effect of all soluble mediators. Alternatively there may be an enhanced tissue uptake and/or clearance of TGF- $\beta$ and it may still have an important biological effect in PVR related fibrogenesis. An additional possibility is that, since our methodology detects total TGF- $\beta$, there may be an increase in biologically active growth factor in the eye which we have not detected and which could enhance PVR development.

The nature and incidence of perisilicone proliferation remain uncertain. Previous reports have documented recurrent epiretinal proliferation behind silicone oil. Lewis et al ${ }^{9}$ found that 19 of 31 eyes $(61 \%)$ developed perisilicone proliferation (and that this led to redetachment in 15 eyes) and Zilis et al reported 21 of 55 eyes (38\%) developed perisilicone proliferation. ${ }^{10}$ In the silicone study, however, there was no difference in prevalence of postoperative macular pucker between eyes randomised to gas or to silicone oil, suggesting that both may have an effect on postoperative epiretinal membrane formation. ${ }^{22}$ The purity of the silicone oil used may have an important role in the incidence of secondary proliferation in these eyes and it has been shown experimentally that contaminated silicone oil has an enhanced effect on retinal pigment epithelium proliferation compared to purified, medical grade silicone oil. ${ }^{23}$ Improvements in the quality of intraocular silicone oils since their initial introduction can therefore be anticipated to result in a decline in the incidence of perisilicone proliferation. Oil purity, however, may not be the only factor leading to a predisposition to secondary membrane formation and the evidence of this study is that, although purified, medical grade silicone oil was used in all cases there remains a concentration effect on potentially fibrogenic mediators.

Despite these caveats silicone oil remains a vital tool in the management of complex retinal detachment. Vitreoretinal surgeons, however, should be aware that its biological effects will alter intraocular physiology and can potentially contribute to enhanced fibrous scarring. In addition, the presence of silicone oil will alter the pharmacodynamics of any adjunctive treatments aimed at modifying the PVR process $^{24}$ potentially producing a concentrated "depot" in the retro-oil fluid.

\section{Authors' affiliations}

R H Y Asaria, C S Sethi, G A Limb, P T Khaw, D G Charteris, Institute of Ophthalmology, Bath Street, London ECIV 9EL, UK

R H Y Asaria, C S Sethi, G A Limb, P T Khaw, D G Charteris, C H Kon, C Bunce, G W Aylward, Moorfields Eye Hospital, City Road, London ECIV 2PD, UK

\section{REFERENCES}

1 Cibis PA, Becker B, Okun E, et al. The use of liquid silicone in retinal detachment surgery. Arch Ophthalmol 1962;68:590-9. 
2 Cibis PA. Recent methods in the surgical treatment of retinal detachment: intravitreal procedures. Trans Ophthalmol Soc UK 1965;85:111-27.

3 Okun E. Intravitreal surgery utilizing liquid silicone. a long term follow up. Trans Pacific Coast Oto-Ophthalmol Soc 1968:49:141-59.

4 Yeo JH, Glaser BM, Michels RG. Silicone oil in the treatment of complicated retinal detachments. Ophthalmology 1987;94:1109-13.

5 Brourman ND, Blumenkranz MS, Cox MS, et al. Silicone oil for the treatment of severe proliferative diabetic retinopathy. Ophthalmology 1989;96:759-64.

6 Andenmatten R, Gonvers M. Sophisticated vitreoretinal surgery in patients with a healthy fellow eye. An 11-year retrospective study. Graefes Arch Clin Exp Ophthalmol 1993;231:495-9.

7 Aylward GW, Cooling RJ, Leaver PK. Trauma-induced retinal detachment associated with giant retinal tears. Retina 1993;13:136-41.

8 Claes C, Zivojnovic R. The use of perfluorocarbon liquids in vitreous surgery Bull Soc Belge Ophtalmol 1990;238: 145-50

9 Lewis H, Burke JM, Abrams GW, et al. Perisilicone proliferation after vitrectomy for proliferative vitreoretinopathy. Ophthalmology 1988;95:583-91

10 Zilis JD, McCuen BW, de Juan E Jr, et al. Results of silicone oil removal in advanced proliferative vitreoretinopathy. Am J Ophthalmol 1989;108:15-21.

11 Nicolai U, Eckardt C. Immunhistochemische befunde von epiretinalen membranen nach silikonolinjektion. Fortschr Ophthalmol 1991;88:660-4

12 Mukai N, Lee PF, Oguri M, et al. A long-term evaluation of silicone retinopathy in monkeys. Can J Ophthalmol 1975;10:391-402.

13 Mukai N, Lee PF, Schepens CL. Intravitreous injection of silicone: an experimental study. II. Histochemistry and electron microscopy. Ann Ophthalmol 1972;4:273-87.

14 Lambrou FH, Burke JM, Aaberg TM. Effect of silicone oil on experimental traction retinal detachment. Arch Ophthalmol 1987;105:1269-72.
15 Machemer R, Aaberg TM, Freeman HM, et al. An updated classification of retinal detachment with proliferative vitreoretinopathy. Am J Ophthalmol $1991 ; 112: 159-65$

16 Lewis GP, Erickson PA, Guerin CJ, et al. Basic fibroblast growth factor : a potential regulator of proliferation and intermediate filament expression in the retina. J Neurosci 1992;12:3968-78.

17 Cassidy L, Barry P, Shaw C, et al. Platelet derived growth factor and fibroblast growth basic levels in the vitreous of patients with vitreoretinal disorders. Br J Ophthalmol 1998:82:181-5.

18 Kon CH, Occleston NL, Aylward GW, et al. Expression of vitreous cytokines in proliferative vitreoretinopathy: a prospective study. Invest Ophthalmol Vis Sci 1999;40:705-12

19 Heij EC, Van de Waarenburg MPH, Blaauwgeers HGT, et al Basic fibroblast growth factor, glutamine synthetase, and interleukin- 6 in vitreous fluid from eyes with retinal detachment complicated by proliferative vitreoretinopathy. Am J Ophthalmol 2002;134:367-75.

20 Limb GA, Earley O, Jones SE, et al. Expression of mRNA for interleukin 6 by cells infiltrating epiretinal membranes in proliferative vitreoretinopathy Graefes Arch Clin Exp Ophthalmol 1994:232:646-51.

21 Limb GA, Little BC, Meager A, et al. Cytokines in proliferative vitreoretinopathy. Eye 1991;5:686-93.

22 Cox MS, Azen SP, Barr CC, et al. Macular pucker after successful surgery for proliferative vitreoretinopathy. Silicone Study report 8. Ophthalmology 1995; 102:1884-91

23 Friberg TR, Verstraeten TC, Wilcox DK. Effects of emulsification, purity and fluorination of silicone oil on human retinal pigment epithelial cells. Invest Ophthalmol Vis Sci 1991;32:2030-4.

24 Asaria RHY, Kon $\mathrm{CH}$, Bunce $\mathrm{C}$, et al. Adjuvant 5-fluorouracil and heparin prevents proliferative vitreoretinopathy: results from a randomised double blind controlled clinical trial. Ophthalmology 2001;108:1179-83. 